\title{
CURRENT DRIVEN INSTABILITY OF A SUB-ALFVÉNIC RELATIVISTIC JET
}

\author{
PHILIP E. HARDEE \\ Department of Physics and Astronomy, The University of Alabama \\ Tuscaloosa, AL 35487, USA \\ phardee@bama.ua.edu \\ YOSUKE MIZUNO \\ Institute for Astronomy, National Tsing-Hua University \\ 101, Sec. 2, Kuang Fu Road, Taiwan 30013, R.O.C. \\ mizuno@phys.nthu.edu.tw \\ KEN-ICHI NISHIKAWA \\ Center for Space Plasma and Aeronomic Research, University of Alabama in Huntsville \\ 320 Sparkman Drive, NSSTC, Huntsville, AL 35805, USA \\ ken-ichi.nishikawa@nsstc.nasa.gov
}

\begin{abstract}
We have investigated the influence of velocity shear on the linear and non-linear development of the CD kink instability. We follow temporal development of the instability within a periodic computational box. We find that helically distorted density structure propagates along the jet with speed and flow structure dependent on the location of the velocity shear relative to the characteristic radius of the helically twisted force-free magnetic field. At small radius the plasma flows through the kink. The kink propagation speed increases as the radius increases and the kink becomes more embedded in the plasma flow. Larger velocity shear radius leads to slower linear growth, makes a later transition to the nonlinear stage, and with larger maximum amplitude than occurs for a static plasma column. However, when the velocity shear radius is much greater than the characteristic radius of the helical magnetic field, linear and non-linear development become more similar to the development of a static plasma column.
\end{abstract}

Keywords: instabilities - MHD - methods: numerical - galaxies: jets

\section{Introduction}

Relativistic jets occur in black hole binary star systems ( $\mu$ QSOs), occur in active galactic nuclei (AGN), can be associated with neutron stars and pulsar wind nebulae, and are thought responsible for the gamma-ray bursts (GRBs). It is thought that these jets are generated hydromagnetically and collimation is generally attributed to the toroidal magnetic field acting in concert with an external pressure. ${ }^{1}$ In general, this configuration is current-driven (CD) kink mode unstable. ${ }^{2-7}$ This instability can strongly distort or even disrupt the system and numerical simulations are needed to study the non-linear development of this instability. 
Twisted structures are observed in AGN jets on sub-parsec, parsec and kiloparsec scales. ${ }^{8-9}$ Numerical simulations have showed structures attributed to CD kink instability, ${ }^{10-17}$ and structures attributed to Kelvin-Helmholtz (KH) velocity shear driven helical instability. ${ }^{18-21}$ It is not clear whether current driven, velocity shear driven or jet precession is responsible for the observed twisted structures at different spatial scales. In any case, the investigation of instability and non-linear stabilization should allow selection between different jet configurations that formally satisfy the magneto-hydrodynamic (MHD) equations.

\section{Numerical Method and Setup}

The RAISHIN simulation code has passed 1D, 2D and 3D relativistic test problems (see Ref. 20). The code also delivers results that agree with both $\mathrm{KH}^{21}$ and $\mathrm{CD}^{22}$ instability problems. Thus, the code reliably solves multidimensional problems.

Here we choose a force-free helical magnetic field. ${ }^{20,22}$ We studied constant and decreasing helical pitch cases but show the constant pitch case here. For constant helical pitch the toroidal component is a maximum at the "characteristic radius" $R_{a}=a$. Our simulation grid of length $L_{z}=12 a$ is periodic in the $z$ direction. The grid is a Cartesian $(x, y, z)$ box of size $4 / 3 L_{z} \times 4 / 3 L_{z} \times L_{z}$ with grid resolution $\Delta L_{z}=$ $L_{z} / 120$. We impose outflow boundary conditions on the transverse boundaries at $x=y= \pm 2 / 3 L_{z}= \pm 8 a$. We consider a low constant gas pressure medium with equation of state $p=(\Gamma-1) \rho e$, where $e$ is the specific internal energy density and the adiabatic index $\Gamma=5 / 3$, and initially $p=p_{0}=0.02 \rho_{0} c^{2}$.

To break the symmetry the initial MHD equilibrium is perturbed by a radial velocity component with helical twist wavelength equal to the box length, amplitude $\delta v=0.01 c$, and with radial width $R_{p}=2 a$. We performed simulations with velocity shear locations $R_{j}=a / 2, a, 2 a$ and $4 a$, and two different velocities. We use a nonuniform density profile decreasing with the magnetic field strength in order to keep the jet sub-Alfvénic inside the velocity shear surface. Such a configuration is CD unstable and $\mathrm{KH}$ stable.

\section{Constant Helical Pitch and $v_{j}=0.2 c$}

The left panels in Figure 1 show the time evolution of the volume-averaged kinetic, $E_{k i n}$, and magnetic, $E_{\text {mag }}$, energy transverse to the $z$-axis within a cylinder of radius $R=4 a$ and indicate the growth of the CD kink wavelength $\lambda=12 a$. The KruskalShafranov criterion indicates instability for $\lambda>2 \pi a$ and for our initial conditions the maximum growth rate occurs at $\lambda_{\max } \approx 10 \mathrm{a}$. The maximum temporal growth rate in the kink rest frame should be reduced in the simulation frame by the kink Lorentz factor. ${ }^{7}$ The evolution of $E_{k i n}$ indicates an initial linear growth phase, i.e., exponential increase by about 3 orders of magnitude, to simulation times $t \sim$ $40-50 t_{s}\left(t_{s} \equiv 4 a / c\right)$ depending on the location of the velocity shear radius. At the smallest and largest velocity shear radius growth is most similar to that of a static plasma column. Slower growth when $R_{j}=4 a$, suggests growth like that of a static plasma column but modified by relativistic effects. 

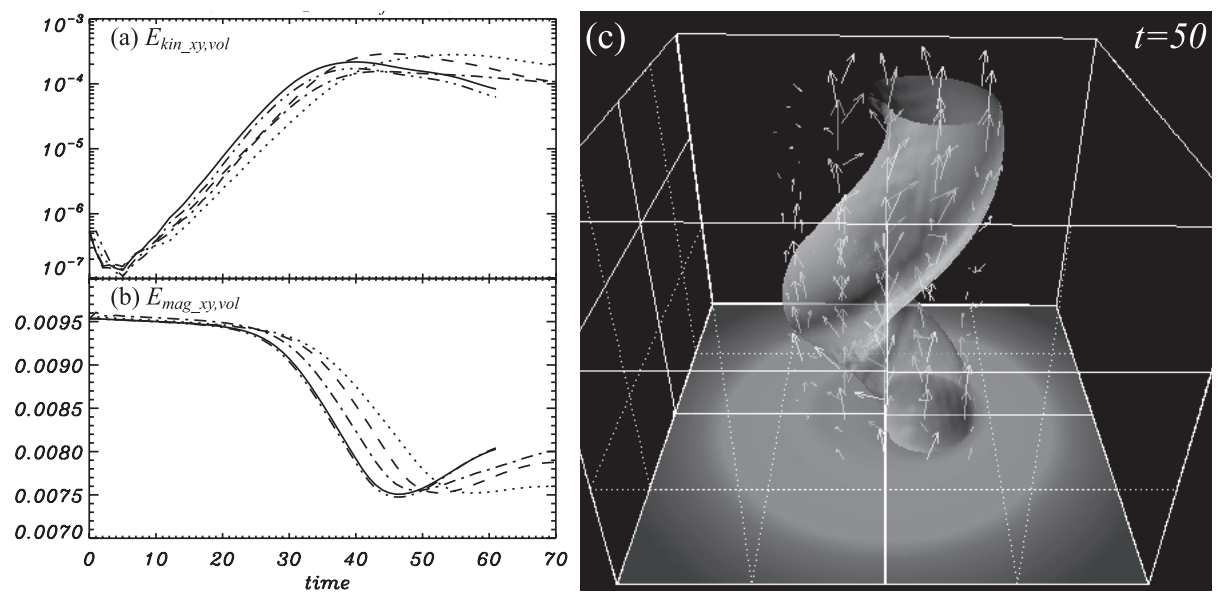

Fig. 1. Left panels: Time evolution of (a) $E_{k i n}$ and (b) $E_{m a g}$ at $R_{j} / a=0.5$ (solid line), 1.0 (dashed line), 2.0 (dotted line) and 4.0 (dash-dotted line). A static plasma column case is shown as a dash-double-dotted line. Right panel (c): Three-dimensional density isosurface at $t=50 t_{s}$ for $R_{j} / a=2.0$ with velocity vectors indicated by the arrows.

The right panel in Figure 1 shows a density isosurface and velocity vectors in the non-linear phase at $t=50 t_{s}$ for $R_{j} / a=2.0$. For this intermediate velocity shear radius the kink propagates along the z-axis but at less than the flow speed and the flow follows the helical twist. At small velocity shear radius the kink is almost stationary with flow through the kink. At larger velocity shear radius the helical kink is embedded within and initially moving with the flow. For larger velocity shear radius, the kink slows as the transverse amplitude of the growing kink approaches the velocity shear radius.

Our results show that kink growth, propagation and flow morphology are strongly dependent on the velocity shear radius relative to the characteristic radius. At one extreme the fluid flows through the kink with helicity comparable to that of the kink and at the other extreme the kink is embedded within a more uniform flow. For velocity shear radii on the order of the characteristic radius there is an intimate interaction between the growing kink and the flow field. In general, the kink propagates more slowly than the flow and slows as the amplitude increases. Thus, the flow field becomes more helically twisted as the kink amplitude increases.

\section{Discussion}

In general, the growth of CD kink instability in the initial exponential growth phase is slower than found for a static plasma column. There is more reduction in the growth rate for constant magnetic pitch than for decreasing magnetic pitch, and more reduction in the growth rate if the velocity shear is larger (see Ref. 20). Thus, decreasing the magnetic pitch or increasing the flow velocity enhances the influence of the velocity shear surface. In the absence of relativistic effects which slow the observed rate of growth, it is clear that the presence of a velocity shear surface has 
the strongest influence on both the linear and non-linear behavior of the growing kink when the velocity shear is located at or outside the characteristic radius.

For the largest velocity shear radius $R_{j}=4 a$ where the kink moves with the flow frame we find an e-folding time in the lab frame significantly longer than the predicted kink Lorentz factor times the e-folding time in the flow frame. ${ }^{7} \mathrm{We}$ speculate that the large increase in e-folding time measured in the lab frame is partly a result of the increased inertia of the relativistically moving fluid. ${ }^{20}$

The 3D relativistic jet generation simulation performed by Ref. 16 indicates relatively rapid, less than 100 gravitational radii, but non-disruptive kink development over 500 gravitational radii. Our previous and present simulations for static and moving kinks suggest that the rapid but non-disruptive kink development in the jet generation simulation could be a result of a velocity shear surface located less or on order of the characteristic magnetic radius.

\section{Acknowledgments}

Work supported by NSF awards AST-0506719, AST-0506666, AST-0908010 \& AST0908040, NASA awards NNG05GK73G, NNX07AJ88G \& NNX08AG83G, Taiwan NSC award 100-2112-M-007-022-MY3, and US-Israeli BSF award 2006170. Simulations performed on Columbia at NASA Ames, and the SGI Altix at the NCSA.

\section{References}

1. R. D. Blandford, Phil. Trans. Roy. Soc. A 358, 1 (2000).

2. Y. N. Istomin and V.I. Pariev, Mon. Not. R. Astron. Soc. 267, 629 (1994).

3. Y. N. Istomin and V.I. Pariev, Mon. Not. R. Astron. Soc. 281, 1 (1996).

4. M. C. Begelman, Astrophys. J. 493, 291 (1998).

5. Y. E. Lyubarskii, Mon. Not. R. Astron. Soc. 308, 1006 (1999).

6. A. Tomimatsu, T. Matsuoka and M. Takahashi, Phys. Rev. D 64, 123003 (2001).

7. R. Narayan, J. Li and A. Tchekhovskoy, Astrophys. J. 697, 1681 (2009).

8. J. L. Gómez et al., Astrophys. J. 561, L161 (2001).

9. A. P. Lobanov, and J. A. Zensus, Science 294, 128 (2001).

10. T. Lery, H. Baty and S. Appl, Astron. Astrophys. 355, 1201 (2000).

11. R. Ouyed, D. A. Clarke and R. E. Pudritz, Astrophys. J. 582, 292 (2003).

12. M. Nakamura and D. L. Meier, Astrophys. J. 617, 123 (2004).

13. M. Nakamura, H. Li and S. Li, Astrophys. J. 656, 721 (2007).

14. R. Moll, H. C. Spruit and M. Obergaulinger, Astron. Astrophys. 492, 621 (2008).

15. R. Moll, Astron. Astrophys. 507, 1203 (2009).

16. J. C. McKinney and R. D. Blandford, Mon. Not. R. Astron. Soc. 394, L126 (2009).

17. C. S. Carey and C. R. Sovinec, Astrophys. J. 699, 362 (2009).

18. P. E. Hardee et al., Astrophys. J. 555, 744 (2001).

19. P. E. Hardee and P. A. Hughes, Astrophys. J. 583, 116 (2003).

20. Y. Mizuno, P. E. Hardee and K.-I. Nishikawa, Astrophys. J. 734, 19 (2011).

21. Y. Mizuno, P. E. Hardee and K.-I. Nishikawa, Astrophys. J. 662, 835 (2007).

22. Y. Mizuno et al., Astrophys. J. 700, 684 (2009). 